\title{
MULTICOLOR LUMINOSITY FUNCTION OF FIELD GALAXIES
}

\author{
A. BRUNELLO, P. SARACCO, B. GARILLI, D. MACCAGNI \\ IFCTR-CNR, Via Bassini 15,20133 Milano,Italy \\ AND \\ G. CHINCARINI \\ Osservatorio Astronomico di Brera, Via Brera 28,20121 Milano, Italy
}

The aim of this programme is to determine the luminosity function of field galaxies in different bands for a unique sample. From the ESP [1] redshift survey of $3344 \mathrm{bj} \leq 19.4$ galaxies, we have extracted a fair subsample and obtained multicolor photometry for $354(11 \%)$ galaxies in the V, R (Johnson) and i (Gunn) filters and for 148 (4\%) in the $\mathrm{K}^{\prime}$ band. Errors on the total magnitudes are smaller than 0.1 in all bands. In order to obtain absolute magnitudes, we applied k-corrections [2] after deriving a rough morphological classification based on color information. Here we present a by product of this programme: the determination of the colorluminosity relationship. The figure shows how the rest-frame $\mathrm{Bj}-\mathrm{K}$ ' color is strongly related to the absolute i magnitude while such correlation is not present using optical colors. If we assume $M_{i}$ is a good mass tracer, the figure indicates that bluer galaxies are the less massive ones.
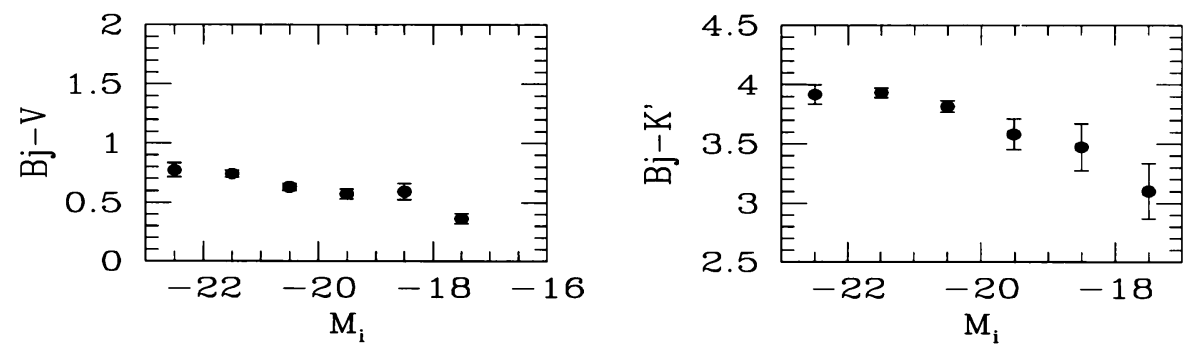

\section{References}

[1] Vettolani, G. et al. (1997) The ESO Slice Project (ESP) galaxy redshift survey. I.Description and first results, $A \& A$, Vol. 325, pp. 954-960

[2] Frei, Z., Gunn, J.E. (1994) Generating colors and k corrections from existing catalog data, $A J$, Vol. 108. II, pp. 1476-1485 